\title{
The influence of prolonged intensive care stay on quality of life, recovery, and clinical outcomes following cardiac surgery: A prospective cohort study
}

Mohammad S. Diab, PhD, MRCS, ${ }^{\text {a }}$ Rajdeep Bilkhu, MRCS, ${ }^{a}$ Gopal Soppa, PhD, FRCS (CTh), ${ }^{a}$ Mark Edsell, FRCA, ${ }^{a}$ Nick Fletcher, FRCA, ${ }^{a}$ Johan Heiberg, MD, PhD,${ }^{\text {b,c }}$ Colin Royse, MD, PhD,${ }^{\text {b,c }}$ and Marjan Jahangiri, FRCS (CTh) ${ }^{\mathrm{a}}$

\section{ABSTRACT}

Objective: To examine the influence of prolonged intensive care unit (ICU) stay on quality of life and recovery following cardiac surgery.

Methods: Quality of life was assessed using the Short Form 36 Health Survey (SF36). The Postoperative Quality of Recovery Scale was used to assess quality of recovery, disability, and cognition after ICU discharge over 12 months' follow-up. Prolonged ICU stay was defined as $\geq 3$ postoperative days. Mortality and major adverse cardiac and cerebrovascular events were recorded up to 12 months.

Results: For quality of life, the physical component improved over time in both groups $(P<.01$ for both groups), as did the mental component $(P<.01$ for both groups). The long ICU group had lower physical and mental components over time (both $P$ values $<.01$ ), but by 12 months the values were similar. The overall quality of recovery was lower for the long ICU group $(P<.01)$. Likewise, we found higher rates of recovery in the normal ICU group than in the long ICU group in terms of emotive recovery $(P<.01)$, activities of daily living $(P<.01)$, and cognitive recovery $(P=.03)$ but no differences in terms of physiologic $(P=.91)$, nociceptive $(P=.89)$, and satisfaction with anesthetic care $(P=.91)$. Major adverse cardiac and cerebrovascular events $(P<.01)$, 30-day mortality $(P<.01)$, and length of ward stay $(P<.01)$ were all higher with prolonged ICU stay.

Conclusions: Patients with prolonged ICU stay have lower quality of life scores; however, they achieve similar midterm quality of recovery, but with reduced survival, increased major adverse cardiac and cerebrovascular events, and longer hospital length of stay. (J Thorac Cardiovasc Surg 2018;156:1906-15)

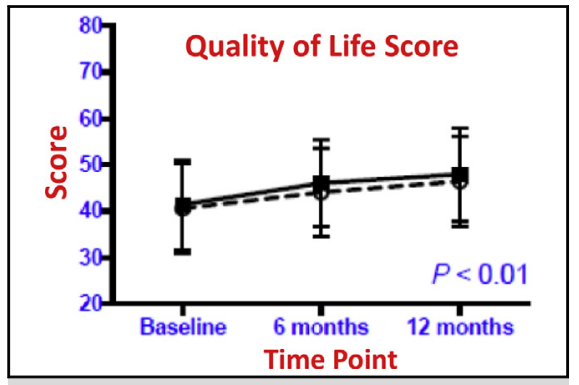

Prolonged ICU stay is associated with inferior QOL outcomes.

\section{Central Message}

Patients surviving long ICU stay ( $\geq 3$ days) following cardiac surgery show a slower but positive trend in $\mathrm{QOL}$ and recovery during midterm follow-up.

\section{Perspective}

Patients experiencing prolonged ICU stay were older with higher cardiac risk profiles, compared with those with shorter ICU stay. The long ICU stay group had poorer QOL and quality of recovery outcomes during follow-up. However, by 12 months both groups showed a similar positive trend of recovery in most domains. The physical, mental, and cognitive recovery of long ICU group survivors is encouraging.

See Editorial Commentary page 1916.

See Editorial page 1905.
Prolonged intensive care unit (ICU) stay is associated with increased mortality, morbidity, and cost of care. ${ }^{1,2}$ There is

\footnotetext{
From the a Departments of Cardiothoracic Surgery, Anaesthesia, and Intensive Care, St George's Hospital, London, United Kingdom; 'bepartment of Anaesthesia, Royal Melbourne Hospital, Melbourne, Victoria, Australia; and ${ }^{\mathrm{c}}$ Department of Surgery, The University of Melbourne, Melbourne, Victoria, Australia.

Funded by St George's Charitable Foundation.

Received for publication May 22, 2017; revisions received May 5, 2018; accepted for publication May 7, 2018.

Address for reprints: Marjan Jahangiri, FRCS (CTh), Department of Cardiac Surgery, St George's Hospital, Blackshaw Rd, London SW17 OQT, United Kingdom (E-mail: marjan.jahangiri@stgeorges.nhs.uk). 0022-5223/\$36.00

Crown Copyright (C) 2018 Published by Elsevier Inc. on behalf of The American Association for Thoracic Surgery

https://doi.org/10.1016/j.jtcvs.2018.05.076
}

paucity of knowledge about quality of life (QOL) amongst ICU survivors.

The number of elderly and high-risk patients undergoing cardiac surgery in the United Kingdom has increased by $5 \%$ to $18 \%$ during the past decade. ${ }^{4,5}$ Similar trends are also seen in the United States and Europe. ${ }^{6,7}$ The

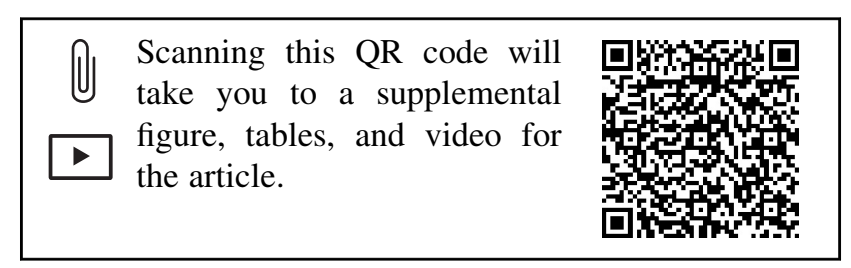




\section{Abbreviations and Acronyms \\ EuroSCORE $=$ European System for Cardiac \\ Operative Risk Evaluation \\ ICU = intensive care unit \\ MACCE $=$ major adverse cardiac and cerebrovascular events \\ MCS = mental component summary \\ PCS = physical component summary \\ PostopQRS = Postoperative Quality of Recovery Scale \\ QOL = quality of life \\ SF36 = Short Form 36 Health Survey}

incidence of prolonged ICU stay following cardiac surgery varies from $4 \%$ to $11 \%$, depending on its definition., ${ }^{4,6,8-10}$ There has been considerable debate on the definition of prolonged ICU stay following surgery, which ranges from $>24$ hours to $>14$ days. ${ }^{8,11,12}$

Due to improved surgical techniques and perioperative care, cardiac surgery mortality has decreased during the past decade, but morbidity and length of ICU stay have not changed. ${ }^{9,10}$ With increasing age and comorbidity of patients undergoing cardiac surgery, an increasing emphasis is placed on QOL after surgery. ${ }^{13,14}$

There are several well-established preoperative risk stratification scoring systems in cardiac surgery, such as the European Score for Cardiac Risk Evaluation (EuroSCORE) and Society of Thoracic Surgeons score, none of which predict prolonged ICU stay or QOL. ${ }^{15,16} \mathrm{~A}$ recent systematic review by our group ${ }^{17}$ concluded that postoperative QOL after prolonged ICU stay following cardiac surgery is variable and that reported QOL outcomes are inconsistent across the reported studies.

The aim of this study was to examine the effect of prolonged ICU stay on QOL outcomes and quality of recovery after cardiac surgery.

\section{METHODS}

\section{Study Design and Patients}

A prospective, single-center longitudinal observational cohort study was carried out. National ethical (institutional review board reference 12/SW/ 0283) and research and design approval (research and design reference 12.0159) were obtained. Consecutive patients undergoing elective and urgent cardiac surgery between October 2013 and September 2015 were invited to participate. Definitions of elective, urgent, and emergency surgery were adopted from The Society for Cardiothoracic Surgery of Great Britain \& Ireland. ${ }^{18}$ Elective operation was defined as the patient being admitted from home for scheduled surgery. Urgent operation was defined when the patient was admitted from another hospital or through the emergency department and could not be discharged home prior to surgery. Patients requiring surgery within 24 hours of admission were defined as emergency and were excluded. These patients could not undergo detailed physical and mental assessment due to their requirement for emergency clinical care. Baseline QOL, clinical, and functional status were assessed before surgery and at several fixed, predetermined time points following discharge from an ICU. Patients undergoing emergency or salvage procedures, those who refused to participate, and those who were unable to give informed consent or did not have adequate comprehension of English were excluded.

\section{Outcomes}

The primary outcomes were QOL and quality of recovery after cardiac surgery. Secondary outcomes were mortality and major adverse cardiovascular and cerebrovascular event (MACCE). Acute kidney injury was diagnosed and staged according to Kidney Disease Improving Global Outcomes guidelines. ${ }^{19}$

\section{Follow-up}

Following cardiac surgery and discharge from ICU, patients were contacted by telephone interview at 14 days and 3 months after discharge and by clinical assessment at 6 and 12 months in a clinic. Questionnaires of QOL and assessment of quality of recovery were administered at these times. For patients who were unable to attend, either a postal survey was sent or a telephone interview was arranged.

\section{QOL Assessment}

QOL was measured using the Short Form 36 Health Survey (SF36). It consists of 36 individual questions, from which a physical component score (PCS) and mental component score (MCS) summary is derived. The final scores of PCS and MCS were calculated using an algorithm (QualityMetrics SF-36v2; RAND Health, Santa Monica, Calif). The population mean score for healthy UK population for the PCS and MCS is 50 and lower score means lower QOL. ${ }^{20}$ The SF36 questionnaire was given to patients before surgery and at 6 and 12 months following ICU discharge after the index procedure. The pre- and postoperative 10 domains of QOL were compared to the UK population norm-based scores (mean, $50 \pm 10$ ). To eliminate inter-observational bias, all questionnaires were administered and followedup by a single researcher.

\section{Recovery Assessment}

Postoperative Quality of Recovery Scale (PostopQRS) is a tool to evaluate postoperative recovery following surgery and anesthesia. ${ }^{21}$ Most current research tools are designed to assess short-term recovery and are not purposely designed to measure recovery over multiple time periods. $^{22,23}$ The PostopQRS was designed to assess individual patient recovery outcomes at multiple time points. It includes 6 domains of recovery (physiologic, nociceptive, emotive, activity of daily living, cognitive, and overall patient perspective). Recovery was defined as return to baseline scores (presurgery) or an improvement from baseline. Assessment was made at baseline, 1 to 14 days before surgery as well as at 1,3 , and 14 days and at 3, 6, and 12 months after ICU discharge either via face-to-face interview or via telephone. An example of the PostopQRS is shown as Online Data Supplement 1.

\section{ICU Discharge Criteria}

Discharge from ICU was determined by predefined, standardized clinical criteria based on clinical decisions and not on bed availability. The patient was discharged from ICU when the patient physiologic state had stabilized, following review by a consultant intensivist, and satisfying the following criteria: extubated and with good gas exchange with minimal supplemental oxygen, alert and orientated, mean arterial pressure $>65 \mathrm{~mm}$ $\mathrm{Hg}$ and on no inotropes, urine output $>0.5 \mathrm{~mL} / \mathrm{kg} / \mathrm{h}$, and no acute kidney injury on the first day.

\section{Definition of Prolonged ICU Stay}

There is no clear definition of prolonged ICU stay following cardiac surgery. ${ }^{17}$ At our unit, and also based on National Cardiac Benchmarking Collaborative data, the majority $(75 \%)$ of patients undergoing cardiac surgery stay in ICU for $<2$ days. ${ }^{24}$ Therefore, the definition of prolonged 


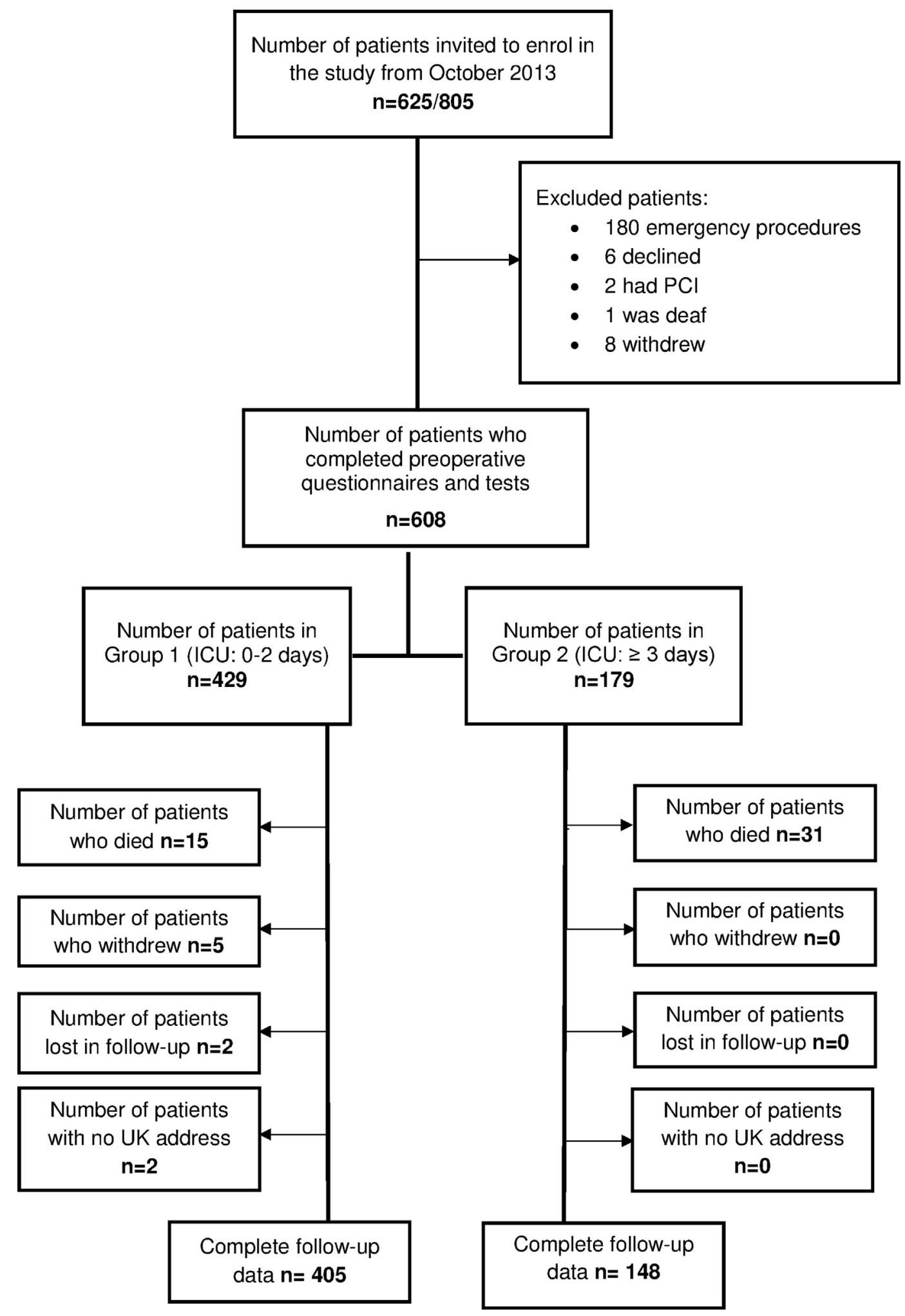

FIGURE 1. Flow of patients through the study period. $P C I$, Percutaneous coronary intervention; $I C U$, intensive care unit; $U K$, United Kingdom. 
TABLE 1. Characteristics of patients undergoing cardiac surgery

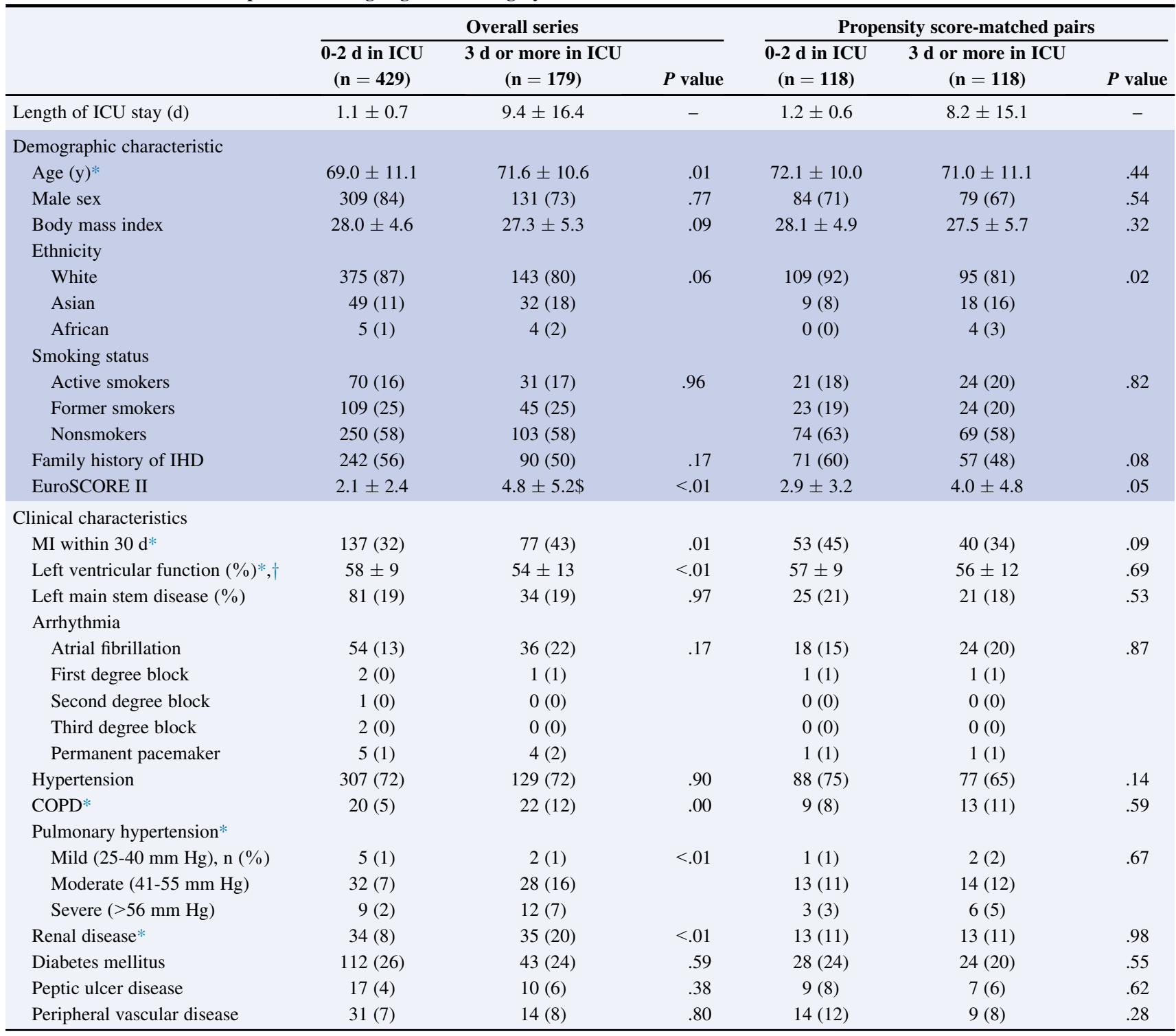

Data reported for the overall series of patients and for the propensity score-matched pairs. Values are presented as means \pm standard deviations or absolute $\mathrm{n}(\%)$. ICU, Intensive care unit; IHD, ischemic heart disease; EuroSCORE, European System for Cardiac Operative Risk Evaluation; $M I$, myocardial infarction; COPD, chronic obstructive pulmonary disease. *Included in the regression model to calculate propensity scores. $\nmid$ Left main stem disease refers to stenosis of more than $50 \%$, and renal function is defined according to the RIFLE (Risk, Injury, Failure, Loss of kidney function, and End-stage kidney disease) criteria.

ICU stay for this study was based on a stay longer than 2 days. Part days were counted as full days, commencing with the first day after surgery (first postoperative day) until discharge from the ICU.

\section{Statistical Analyses}

Patients were divided into 2 groups: ICU stay 0 to 2 days (normal ICU) and ICU stay $\geq 3$ days (long ICU). Binary data were compared using $\chi^{2}$ test and continuous data were checked for normality using histograms and QQ-plots and were compared using independent $t$ tests or Mann-Whitney tests, as appropriate. Differences in QOL were assessed using repeated analyses of variance and differences in quality of recovery were analyzed with general linear mixed model for the analysis of interaction effects (time $\times$ group) (main effects) on PostopQRS recovery status with groups compared using the Cochrane-Mantel-Haentzel test. The long ICU group was subdivided into intervals of 3 to 4 days, 5 to 6 days, and $>6$ days.
Data for this division are displayed as descriptive data only and were not analyzed due to risk of type II error with a small sample size for each subgroup. In the analyses of clinical outcomes, propensity matching was performed, which included all the demographic variables that were significantly different between the 2 groups with a significance level of $P=.05$ (excluding EuroSCORE II). A caliper of 0.005 standard deviations of propensity scores, nearest neighbor matching (1:1), and no replacement was chosen to ensure each group had the same number of patients. Statistical analyses were performed using SPSS (IBM-SPSS Inc, Armonk, NY) version 22 and STATA (StataCorp, College Station, Tex).

\section{RESULTS}

\section{Patients}

Between October 2013 and September 2015, 625 consecutive patients were recruited. After exclusion, 608 patients 
TABLE 2. Operative details

\begin{tabular}{|c|c|c|c|c|c|c|}
\hline \multirow[b]{2}{*}{ Type of surgery* } & \multicolumn{3}{|c|}{ Overall series } & \multicolumn{3}{|c|}{$\begin{array}{c}\text { Propensity score-matched pairs } \\
\end{array}$} \\
\hline & $\begin{array}{l}\overline{0-2} \text { d I ICU } \\
(\mathrm{n}=429)\end{array}$ & $\begin{array}{c}3 \mathrm{~d} \text { or more in ICU } \\
(\mathrm{n}=179)\end{array}$ & $P$ value & 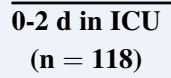 & $\begin{array}{c}3 \mathrm{~d} \text { or more in ICU } \\
(\mathrm{n}=118)\end{array}$ & $P$ value \\
\hline Coronary artery bypass grafting & $222(52)$ & $70(40)$ & $<.01$ & $53(45)$ & $48(41)$ & .98 \\
\hline Valve surgery & $56(13)$ & $10(6)$ & & $8(7)$ & $8(7)$ & \\
\hline Combined procedures & $20(5)$ & $6(3)$ & & $5(4)$ & $6(5)$ & \\
\hline Aortic surgery & $88(21)$ & $60(34)$ & & $34(29)$ & $35(30)$ & \\
\hline Other & $43(19)$ & $33(18)$ & & $18(15)$ & $20(17)$ & \\
\hline Cardiopulmonary bypass time ( $\mathrm{min})^{*}$ & $109 \pm 48$ & $143 \pm 68$ & $<.01$ & $126 \pm 61$ & $126 \pm 57$ & .95 \\
\hline
\end{tabular}

Data reported for the overall series of patients and for the propensity score-matched pairs. Values are presented as absolute $\mathrm{n}(\%)$ or mean \pm standard deviation. ICU, Intensive care unit. *Included in the regression model to calculate propensity scores.

were studied (Figure 1). Of the 608 patients enrolled in the study, 562 patients were followed-up postoperatively, and follow-up was complete at all time points for 532 patients. Of 608 patients, 429 patients were in the normal ICU group, whereas 179 patients were in the long ICU group, which could be further subdivided into intervals of 3 to 4 days $(\mathrm{n}=95), 5$ to 6 days $(\mathrm{n}=31)$, and $>6$ days $(\mathrm{n}=53)$.

\section{Baseline Characteristics and Operative Data}

The baseline characteristics and operative details are shown in Table 1. The median age for all patients was 71.8 years with $72.4 \%$ men. The long ICU group had more preoperative comorbidities, including chronic obstructive pulmonary disease, pulmonary hypertension, renal disease, recent myocardial infarction, and impaired left ventricular function resulting in a higher EuroSCORE II (normal ICU, $2.1 \pm 2.4$; long ICU, $4.8 \pm 5.2 ; P<.01$ ). Operative details are shown in Table 2, and as seen, the long ICU group had a higher proportion of aortic surgery and longer crossclamp and cardiopulmonary bypass times.

\section{QOL}

All patients completed the baseline SF36 questionnaire resulting in $608(100 \%)$ complete preoperative baseline SF36 questionnaires. The reasons for attrition during follow-up are shown in Table E1. During the 12-month follow-up period, $46(7.6 \%)$ patients died. In addition, 2 patients withdrew $(0.3 \%)$, and $32(6.3 \%)$ were lost to follow-up at 6 and 12 months. Five hundred thirty $(94.3 \%)$ and $528(93.9 \%)$ patients, at 6 and 12 months, respectively, completed the questionnaire and were included in the final data analysis.

QOL over time in the normal and long ICU groups, including the breakdown of the long ICU group into 3 to 4,5 to 6 , and $>6$ days, is shown in Figure 2. The physical component improved over time in both groups $(P<.01$ for both groups) as did the mental component $(P<.01$ for both groups). The long ICU group had lower physical and mental components over time (both $P$ values $<.01$ ), but by 12 months the values were similar. There was an association with increased ICU stay and lower QOL scores in both domains. There were significant differences over time in physical functioning $(P<.01)$, role physical $(P<.01)$, general health $(P<.01)$, vitality $(P<.01)$, social functioning $(P<.01)$, role emotional $(P<.01)$, and mental health $(P<.01)$ but not for bodily pain $(P=.18)$ (see Figure E1).

\section{Quality of Recovery}

Overall quality of recovery over time in the normal and long ICU groups, including the breakdown of the long ICU group into 3 to 4,5 to 6 , and $>6$ days, is seen in Figure 3 . The overall quality of recovery was lower for the long ICU group $(P<.01)$, and there was an association with worse recovery for participants having longer ICU stays ( $\geq 5$ days). Likewise, we found higher rates of recovery in the normal ICU than in the long ICU group in terms of emotive recovery $(P<.01)$, activities of daily living $(P<.01)$, and cognitive recovery $(P=.03)$ but no differences in terms of physiological $(P=.91)$, nociceptive $(P=.89)$, and satisfaction with anesthetic care $(P=.91)$ as shown in Figure 4. The predicted and back-transformed means on the PostopQRS using the general linear mixed model for the analysis of interaction effects (time $\times$ group) (main effects) are shown in Tables E2 and E3, respectively.

\section{Clinical Outcomes}

Clinical outcomes at 1 year for the overall series and propensity-matched cohorts are shown in Table 3, and Kaplan-Meier survival curves are shown in Figure 5. In both the overall series and propensity-matched analysis, the incidence of major advance cardiac events, MACCE, death, acute kidney injury, and length of stay were higher in the long ICU group, whereas there was no difference in the incidence of nonatrial fibrillation arrhythmias. In-hospital mortality was higher for the long ICU group $(11.7 \% ; 95 \%$ confidence interval $[\mathrm{CI}], 83.6-93 ; P<.01)$, compared with $1.4 \%$ in the normal ICU group $(95 \% \mathrm{CI}$, 97.8-99.8). Survival to 12 months was lower for the long ICU group $(82.8 \% ; 95 \%$ CI, 77.3-88.3), compared with $96.7 \% \quad(95 \%$ CI, 94.9-98.5). Within the long ICU 
Physical component summary

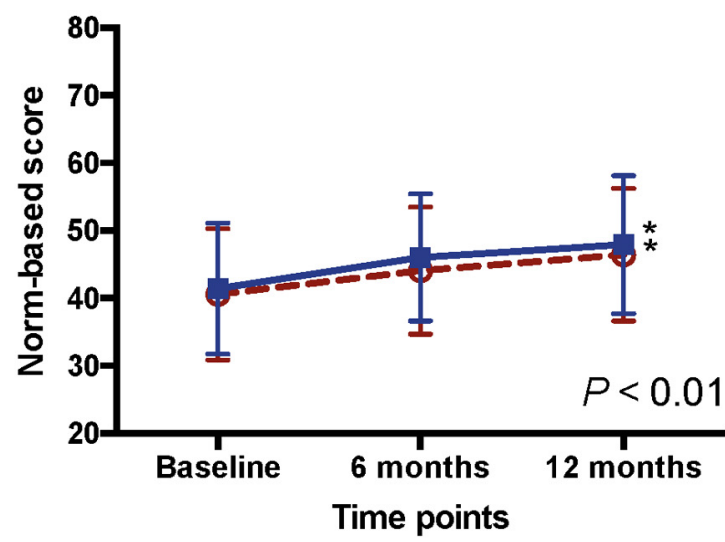

A

NormICU - 5 LonglCU
Physical component summary

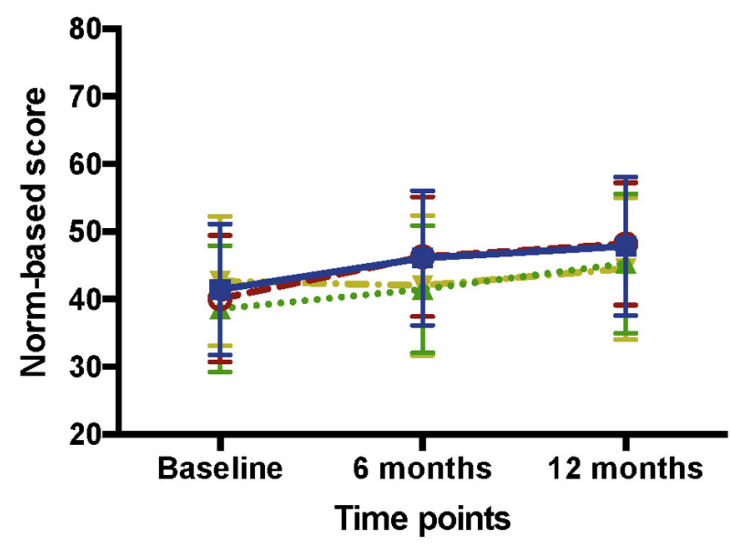

B

\section{Mental Component Summary}

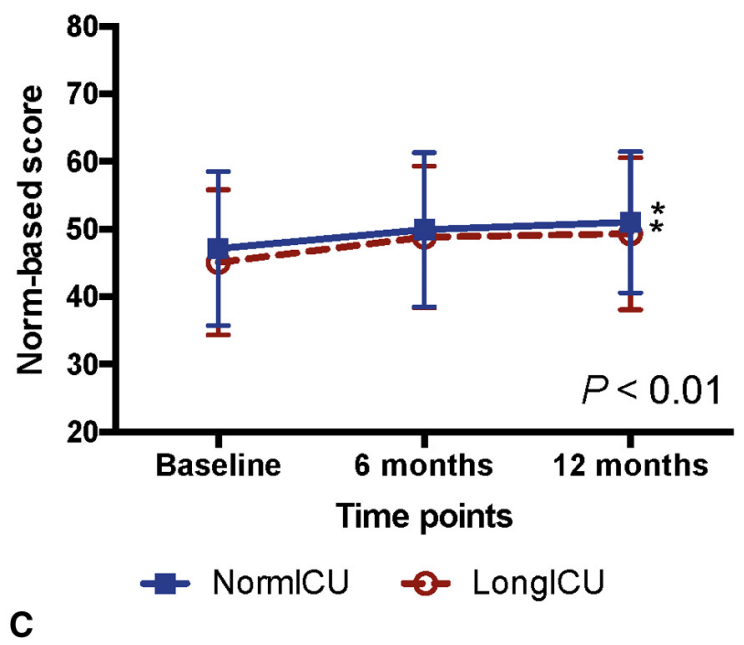

\section{Mental component summary}

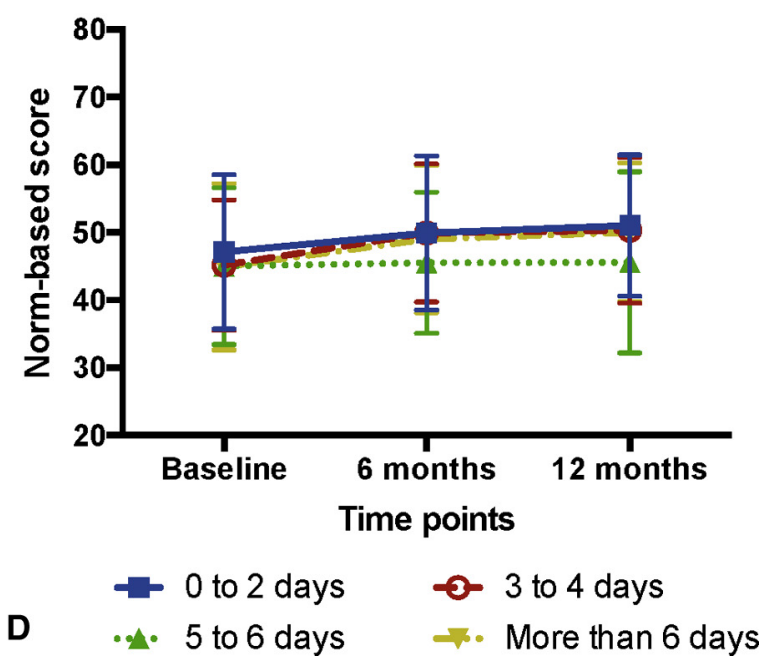

FIGURE 2. Quality of life at baseline, 6 months, and 12 months after surgery, comparing intensive care unit (ICU) length of stay 0 to 2 days (NormICU), and $\geq 3$ days (LongICU). A, Physical component summary. B, Physical component for the LongICU group with subdivision by 3 to 4,5 to 6 , and $>6$ days. C, Mental component summary. D, Mental component summary for the LongICU group with subdivision by 3 to 4,5 to 6 , and $>6$ days. $* P<.05$ for within-group repeated measures analysis of variance analysis, and $\mathrm{P}$ is the value for repeated measures analysis of variance between group analysis over time with Greenhouse Geisser correction. NormICU, Normal intensive care unit stay; LongICU, long intensive care unit stay.

subgroups of 3 to 4 days (95\% CI, 86.2-97.2), 5 to 6 days (95\% CI, 90.5-103.1), and >6 days (95\% CI, 65.1-88.7), there was an association toward further reduced survival only in the $>6$ days subgroup. The incidence of complications for the whole cohort is shown in Table E4.

\section{DISCUSSION}

This study shows that prolonged ICU stay is associated with reduced QOL and a lower incidence of quality of recovery. However, by 12 months the recovery profiles were very similar to the normal ICU group, or had a similar positive trajectory, indicating recovery continues in both groups of patients, even though the long ICU patients experienced more complications. There is an association between longer ICU stay and worse recovery, but even in these subgroups, recovery continued to improve to 12 months.

These results are in accordance with the only prospective study reported on this topic. ${ }^{6}$ Gaudino and colleagues ${ }^{6}$ used the Karnofsky score as the tool for assessing QOL. However, Karnofsky score is not designed for investigating QOL and therefore their results relating to this may not be 


\section{Overall recovery}

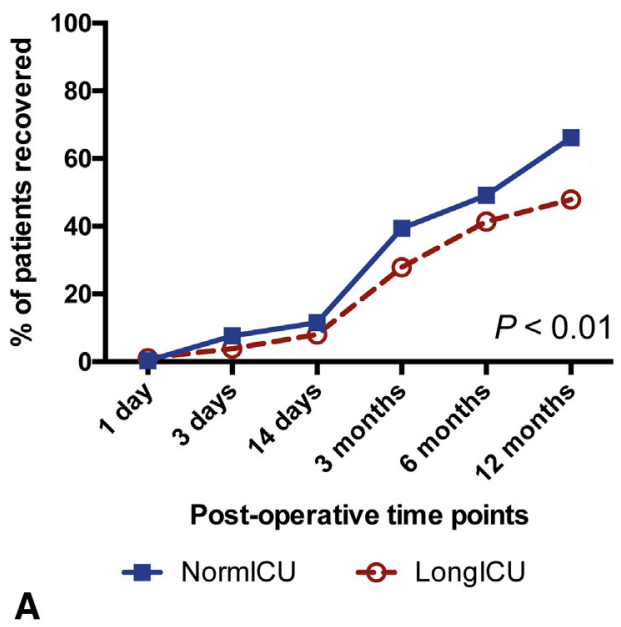

Overall recovery

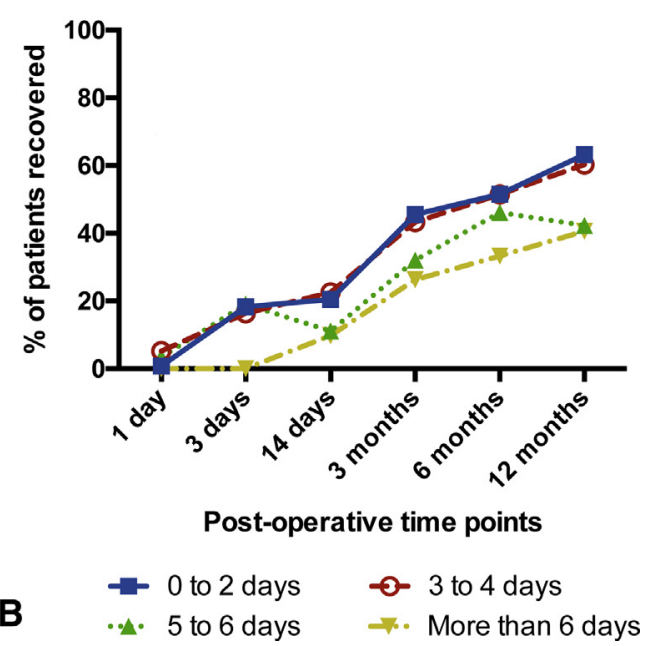

FIGURE 3. Overall quality of recovery over time using the Postoperative Quality of Recovery Scale comparing intensive care unit (ICU) length of stay 0 to 2 days (NormICU), and $\geq 3$ days (LongICU). A, Overall quality of recovery. B, Overall quality of recovery for the LongICU group with subdivision by 3 to 4,5 to 6 , and $>6$ days. $P$ is the value for generalized linear mixed model analysis for between-group analysis over time. NormICU, Normal intensive care unit stay; LongICU, long intensive care unit stay.

justified. In our study, baseline QOL and mean physical component was inferior to the UK normal population, whereas the mental component was only marginally inferior. However, both ICU stay groups showed improvement from baseline in both physical and mental components of QOL at 6 and 12 months' follow-up, with the mean value approaching the UK population average.

Despite the focus on mortality outcomes in cardiac surgery, QOL is considered an increasingly important outcome measure; particularly given the general reduction in mortality following cardiac surgery. ${ }^{25,26}$ Some groups have proposed using QOL outcomes as an indicator to compare cardiac center performances. Moreover, the American College of Cardiology and the American Heart Association guidelines for coronary artery bypass graft surgery have incorporated QOL outcome as a primary indicator for this treatment modality. ${ }^{25}$ One study on patients undergoing CABG showed that low baseline physical component was the only predictor of poor physical at follow-up. ${ }^{26}$

Overall quality of recovery was lower in the long ICU group, but showed a linear improvement by 12 months. Postoperative cognitive decline has been reported to occur in $30 \%$ to $80 \%$ of patients following cardiac surgery. ${ }^{27}$ Although patients with long ICU stay showed slower cognitive recovery, compared with patients with normal ICU stay, the recovery profiles overlapped by 3 months and showed no difference in cognitive recovery thereafter. This finding was also supported by 1 other study. ${ }^{27}$ The recovery profiles for the activities of daily living and emotive domains remained separate. It is, however, reassuring for patients and clinicians that the quality of recovery continues to improve with time.

There is only 1 study in the literature that examines the correlation between the rate of recovery and QOL following cardiac surgery, ${ }^{28}$ but there is no existing research on quality of recovery in relation to ICU discharge after cardiac surgery. Furthermore, there is no clear definition of prolonged ICU stay in the literature, as highlighted in a recent systematic review by our group. ${ }^{17}$ However, ICU stay of $\geq 3$ days is used in 6 other studies. ${ }^{16,29-33}$ In the current study, $\geq 3$ days was chosen as the cutoff point based on local ICU stay duration. Our finding of an increase in MACCE from day 2 to day 3 of ICU stay supports this. Similarly, Silberman and colleagues ${ }^{34}$ found that outcomes were worse when ICU stay exceeded 2 days.

We performed a retrospective pilot study before commencing this current study. Analysis of a cohort of 2250 patients who underwent cardiac surgery was undertaken to establish the clinical relevance and feasibility. ${ }^{35}$ It was demonstrated that patients who had a prolonged ICU stay had higher early and late mortality, although survivors had a satisfactory functional status at 1 year. The present study also supports previous findings that prolonged ICU stay is associated with higher morbidity and mortality. ${ }^{4,8,9,16}$ To reduce the confounder bias for mortality and morbidity outcomes we performed propensity matching. However, mortality in the long ICU remained higher than the normal ICU group. This is not surprising because prolonged ICU may be a consequence 


\section{Physiological recovery}

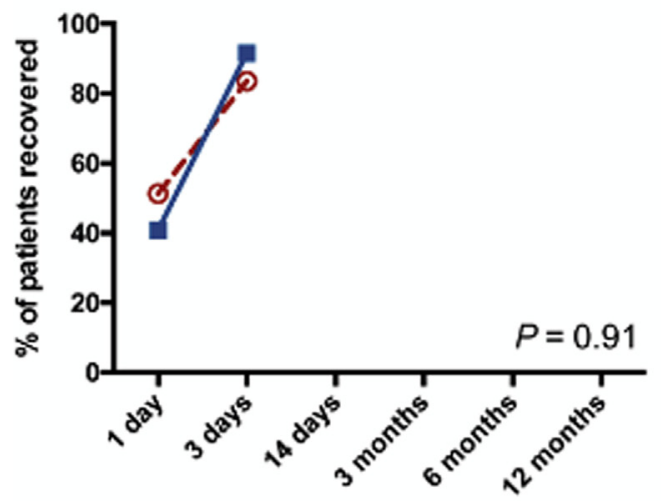

Post-operative time points

$$
\text { A } \quad-\text { NormICU } \nrightarrow \text { LongICU }
$$

\section{Emotive recovery}

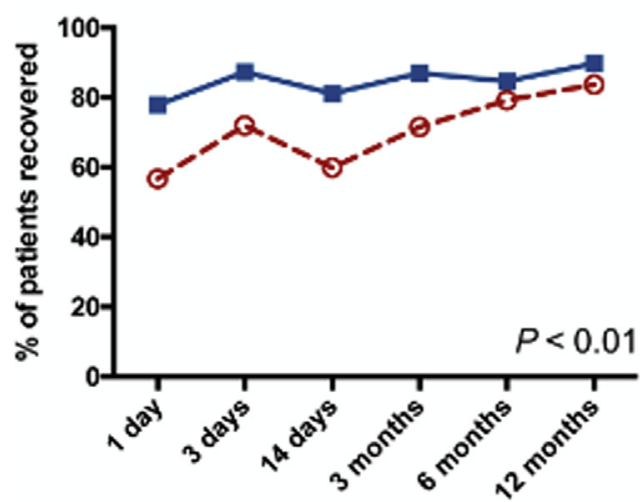

Post-operative time points

C $\rightarrow$ NormlCU $\nrightarrow$ - LonglCU

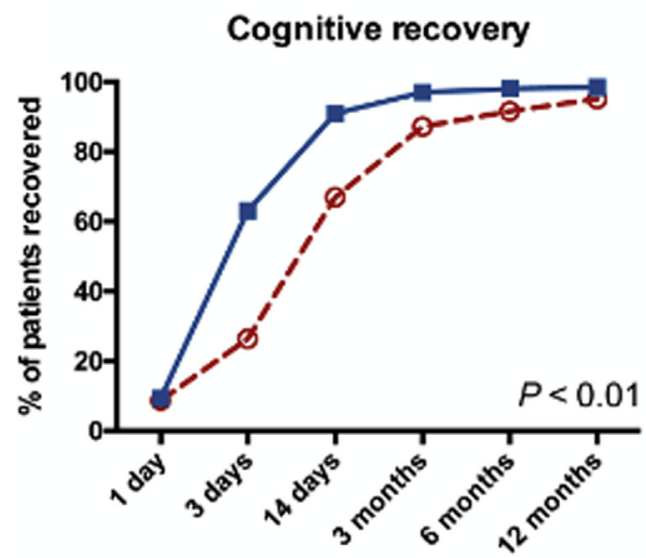

Post-operative time points

$$
\text { E } \quad-\text { NormICU LongICU }
$$

\section{Nociceptive recovery}

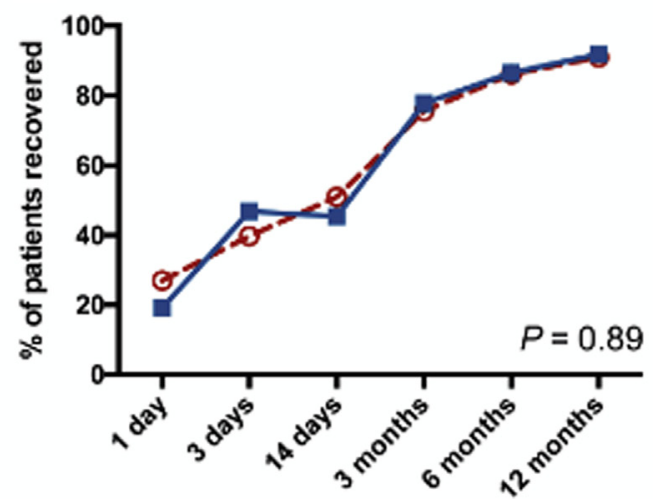

Post-operative time points

B $\quad-$ NormICU $\rightarrow$ LongICU

ADL recovery

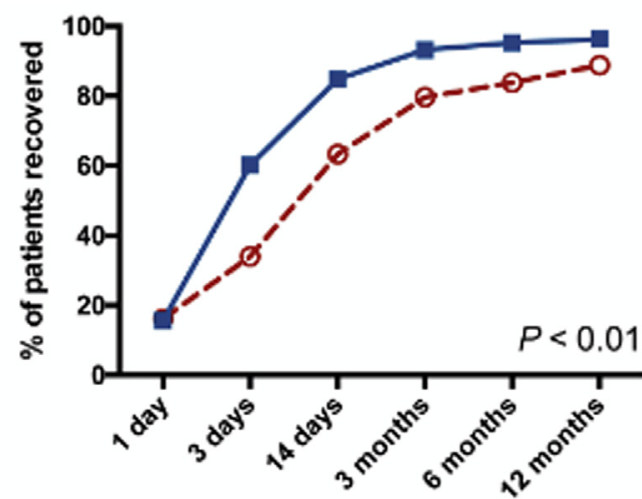

Post-operative time points

D $\quad-$ NormiCU $\rightarrow$ - LonglCU

Satisfaction with anaesthetic care

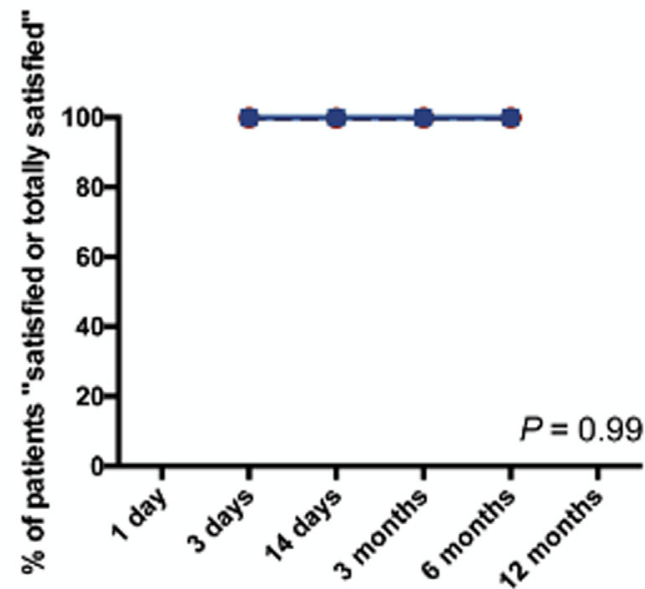

Post-operative time points

NormICU

- LongICU

FIGURE 4. Quality of recovery for individual recovery domains over time using the Postoperative Quality of Recovery Scale comparing intensive care unit (ICU) length of stay 0 to 2 days (NormICU), and $\geq 3$ days (LongICU). A, Physiologic recovery. B, Nociceptive recovery. C, Emotive recovery. D, Activities of daily living (ADL) recovery. E, Cognitive recovery. F, Satisfaction with anesthetic care. $P$ is the value for generalized linear mixed model analysis for between-group analysis over time. NormICU, Normal intensive care unit stay; LongICU, long intensive care unit stay. 
TABLE 3. Clinical outcomes 1 year after cardiac surgery

\begin{tabular}{|c|c|c|c|c|c|c|}
\hline & \multicolumn{3}{|c|}{ Overall series } & \multicolumn{3}{|c|}{ Propensity score-matched pairs } \\
\hline & 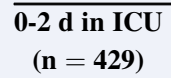 & $\begin{array}{c}3 \mathrm{~d} \text { or more in ICU } \\
(\mathrm{n}=179)\end{array}$ & $P$ value & $\begin{array}{c}\overline{0-2} \mathrm{~d} \text { in } \mathrm{ICU} \\
(\mathrm{n}=118)\end{array}$ & $\begin{array}{c}3 \mathrm{~d} \text { or more in ICU } \\
(\mathrm{n}=118)\end{array}$ & $P$ value \\
\hline MACE & $49(8)$ & $69(25)$ & $<.01$ & $13(11)$ & $43(36)$ & $<.01$ \\
\hline MACCE & $60(11)$ & $80(34)$ & $<.01$ & $19(16)$ & $49(42)$ & $<.01$ \\
\hline Death & $15(3)$ & $31(17)$ & $<.01$ & $6(5)$ & $15(13)$ & .04 \\
\hline Acute kidney injury & $28(7)$ & $66(37)$ & $<.01$ & $7(6)$ & $35(30)$ & $<.01$ \\
\hline Arrhythmias (non-AF) & $19(4)$ & $11(6)$ & .37 & $7(6)$ & $6(5)$ & .79 \\
\hline Length of ward stay (d) & $9 \pm 7$ & $24 \pm 29$ & $<.01$ & $9 \pm 7$ & $22 \pm 26$ & $<.01$ \\
\hline Total length of stay (d) & $10 \pm 7$ & $33 \pm 42$ & $<.01$ & $10 \pm 7$ & $30 \pm 37$ & $<.01$ \\
\hline
\end{tabular}

Data reported for the overall series of patients and for the propensity score-matched pairs. Values are presented as absolute n (\%) or mean \pm standard deviation. ICU, Intensive care unit; $M A C E$, major adverse cardiac event, including nonfatal myocardial infarction, percutaneous coronary intervention, and coronary artery bypass grafting; $M A C C E$, major adverse cardiac and cerebrovascular event, including nonfatal myocardial infarction, percutaneous coronary intervention, coronary artery bypass grafting, and stroke; $A F$, atrial fibrillation.

of high preoperative morbidity, complex surgery, or perioperative complications rather than the cause.

The main limitation of this study was the relatively short follow-up period of 1 year. The measures of QOL may change further with longer follow-up. Another limitation is the lack of patients undergoing heart transplantation or assist devices in this center. These patients usually constitute a large patient cohort that spends an extended time in ICUs. We have shown face validity that outcomes are likely to be worse as the length of ICU stay increases. However, due to the smaller sample size of the subgroups we did not perform statistical analysis due to risk of type II error.

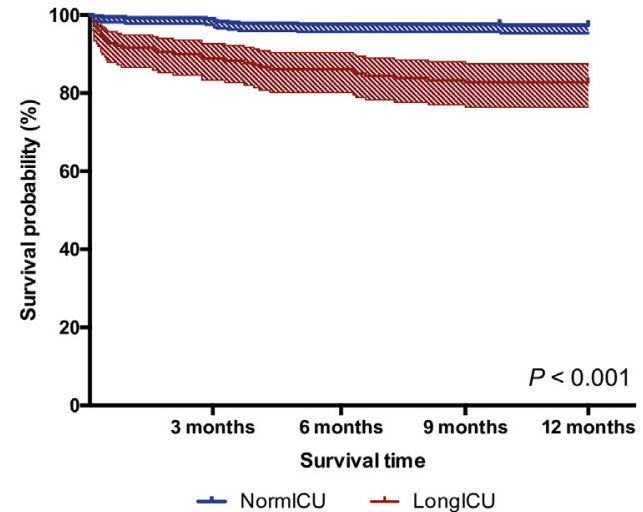

\begin{tabular}{llllll} 
NormICU & 429 & 421 & 415 & 414 & 413 \\
LongICU & 179 & 159 & 154 & 150 & 149 \\
\cline { 2 - 5 } & \multicolumn{5}{c}{ Number of patients at risk }
\end{tabular}

A

FIGURE 5. Kaplan-Meyer curves for survival to 12 months. A, Survival comparison between intensive care unit (ICU) length of stay 0 to 2 days (NormICU) and $\geq 3$ days (LongICU). B, Survival for the LongICU group with subdivision by 3 to 4,5 to 6 , and $>6$ days. NormICU, Normal intensive care unit stay; LongICU, long intensive care unit stay. 


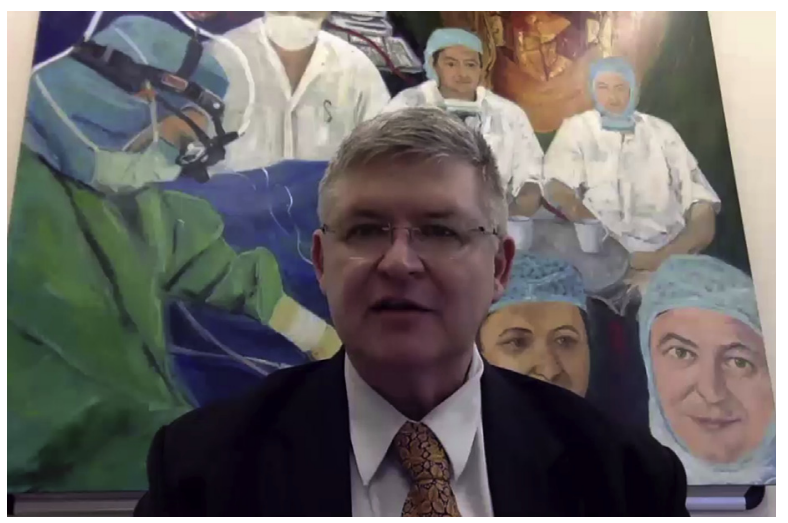

VIDEO 1. A video summary of the key finding of the study. Video available at: https://www.jtcvs.org/article/S0022-5223(18)31506-X/fulltext.

\section{Conflicts of Interest Statement}

Authors have nothing to disclose with regard to commercial support.

\section{References}

1. Zilberberg MD, Luippold RS, Sulsky S, Shorr AF. Prolonged acute mechanical ventilation, hospital resource utilization, and mortality in the United States. Crit Care Med. 2008;36:724-30.

2. Stricker K, Rothen HU, Takala J. Resource use in the ICU: short- vs. long-term patients. Acta Anaesthesiol Scand. 2003;47:508-15.

3. Oeyen SG, Vandijck DM, Benoit DD, Annemans L, Decuyenaere JM. Quality of life after intensive care: a systematic review of the literature. Crit Care Med. 2010;38:2386-400.

4. Bapat V, Allen D, Young C, Roxburgh J, Ibrahim M. Survival and quality of life after cardiac surgery complicated by prolonged intensive care. J Card Surg. 2005;20:212-7.

5. Van Mastrigt GAPG, Heijmans J, Severens JL, Fransen EJ, Roekaerts P, Voss G, et al. Short-stay intensive care after coronary artery bypass surgery: randomized clinical trial on safety and cost-effectiveness. Crit Care Med. 2006;34:65-75.

6. Gaudino M, Girola F, Piscitelli M, Anselmi A, Dell Vella C, Schiavello R, et al. Long-term survival and quality of life of patients with prolonged postoperative intensive care unit stay: unmasking an apparent success. J Thorac Cardiovasc Surg. 2007;134:465-9.

7. Sandroed O, Klepstad P, Stenseth R, Gjeilo KH. Survival and health-related quality of life in cardiac surgery patients with ICU length of stay more than one day. A 5year follow-up study. Eur J Cardiovasc Nurs. 2014;13(Suppl):S71-2.

8. Williams MR, Wellner RB, Hartnett EA, Thornton B, Kavarana MJ, Mahapatra R, et al. Long-term survival and quality of life in cardiac surgical patients with prolonged intensive care unit length of stay. Ann Thorac Surg. 2002; 73:1472-8.

9. Bashour CA, Yared JP, Ryan TA, Rady MY, Mascha E, Leventhal MJ, et al. Long-term survival and functional capacity in cardiac surgery patients after prolonged intensive care. Crit Care Med. 2000;28:3847-53.

10. Gersbach P, Tevaearai H, Revelly JP, Bize P, Ciolero R, von Segesser LK. Are there accurate predictors of long-term vital and functional outcomes in cardiac surgical patients requiring prolonged intensive care? Eur J Cardiothorac Surg. 2006;29:466-72.

11. Kurki TS. Prediction of outcome in cardiac surgery. Mount Sinai J Med. 2002;69: 68-72.

12. Heimrath OP, Buth KJ, Legare JF. Long-term outcomes in patients requiring stay of more than 48 hours in the intensive care unit following coronary bypass surgery. J Crit Care. 2007;22:153-8.

13. Engoren M, Buderer NF, Zacharias A. Long-term survival and health status after prolonged mechanical ventilation after cardiac surgery. Crit Care Med. 2008;28: 2742-9.

14. Clough RA, Leavitt BJ, Morton JR, Plume SK, Hernandez F, Nugent W, et al. The effect of comorbid illness on mortality outcomes in cardiac surgery. Arch Surg. 2002; 137:428-32.
15. Higgins TL. Quantifying risk and assessing outcome in cardiac surgery J Cardiothorac Vasc Anesth. 1998;12:330-40.

16. Ghotkar SV, Grayson AD, Dihmis WC. Effect of prolonged intensive care stay on survival following coronary surgery. Asian Cardiovasc Thorac Ann. 2005;13: 345-50.

17. Diab M, Bilkhu R, Soppa G, McGale N, Hirani SP, Newman SP, et al. Quality of life in relation to length of intensive care unit stay after cardiac surgery. J Cardiothorac Vasc Anesth. 2017;31:1080-90.

18. The Society for Cardiothoracic Surgery in Great Britain \& Ireland. Blue book online. Available at: www.bluebook.scts.org. Accessed June 9, 2018.

19. Improving Global Outcomes Acute Kidney Injury Work Group. KDIGO clinical practice guideline for acute kidney injury. Kidney Int. 2012;2(Suppl): $1-138$.

20. Jenkinson C, Stewart-Brown S, Petersen S, Paice C. Assessment of the SF-36 version 2 in the United Kingdom. J Epidemiol Community Health. 1999;53: 46-50.

21. Royse CF, Newman S, Chung F, Stygall J, McKay RE, Boldt J, et al Development and feasibility of a scale to assess postoperative recovery: the post-operative quality recovery scale. Anesthesiology. 2010;113: 892-905.

22. Aldrete JA. The post-anesthesia recovery score revisited. J Clin Anesth. 1995; 89-91.

23. Hogue SL, Reese PR, Colopy M, Fleisher LA, Tuman KJ, Twersky RS, et al Assessing a tool to measure patient functional ability after outpatient surgery Anesth Analg. 2000;91:97-106.

24. National Cardiac Benchmarking Collaborative. Adult cardiac surgery. Available at: http://www.ucl.ac.uk/nicor/audits/adultcardiac. Accessed April $15,2017$.

25. Eagle KA, Guyton RA, Davidoff R, Edwards FH, Ewy GA, Gardner TJ, et al ACC/AHA 2004 guideline update for coronary artery bypass graft surgery: a report of the American College of Cardiology/American Heart Association task force on practice guidelines (Committee to Update the 1999 Guidelines for Coronary Artery Bypass Graft Surgery). Circulation. 2004;110: e340-437.

26. Le Grande MR, Elliott PC, Murphy BM, Worcester MU, Higgins RO, Ernerst CS, et al. Health related quality of life trajectories and predictors following coronary artery bypass surgery. Health Qual Life Outcomes. 2006;4:49.

27. Boodhwani M, Rubens F, Wozny D, Rodriguez R, Nathan HJ. Effects of sustained mild hypothermia on neurocognitive function after coronary artery bypass surgery: a randomized, double-blind study. J Thorac Cardiovasc Surg. 2007; 134:1443-50

28. Myles PS, Hunt JO, Fletcher H, Solly R, Woodward D, Kelly S. Relation between quality of recovery in hospital and quality of life at 3 months after cardiac surgery. Anesthesiology. 2001;95:862-7.

29. Christakis GT, Fremes SE, Naylor CD, Chen E, Rao V, Goldman BS. Impact of preoperative risk and perioperative morbidity on ICU stay following coronary bypass surgery. Cardiovasc Surg. 1996;4:29-35.

30. Bucerius J, Gummert JF, Walther T, Doll N, Falk V, Schmitt DV, et al. Predictors of prolonged ICU stay after on-pump versus off-pump coronary artery bypass grafting. Intensive Care Med. 2004;30:88-95.

31. Janssen DP, Noyez L, Wouters C, Brouwer RM. Preoperative prediction of prolonged stay in the intensive care unit for coronary bypass surgery. Eur $J$ Cardiothorac Surg. 2004;25:203-7.

32. Hein OV, Birnbaum J, Wernecke KD, Konertz W, Jain U, Spies C. Three-year survival after four major post-cardiac operative complications. Crit Care Med. 2006:34:2729-37.

33. Abrahamyan L, Demirchyan A, Thompson ME, Hovaguimaian H. Determinants of morbidity and intensive care unit stay after coronary surgery. Asian Cardiovasc Thorac Ann. 2006;14:114-8.

34. Silberman S, Bitran D, Fink D, Tauber R, Merin O. Very prolonged stay in the intensive care unit after cardiac operations: early results and late survival. Ann Thorac Surg. 2013;96:15-21.

35. Soppa G, Woodford C, Yates M, Shetty R, Moore M, Valencia O, et al. Functional status and survival after prolonged intensive care unit stay following cardiac surgery. Interact Cardiovasc Thorac Surg. 2013;16:750-4.

Key Words: quality of life, outcomes, recovery 

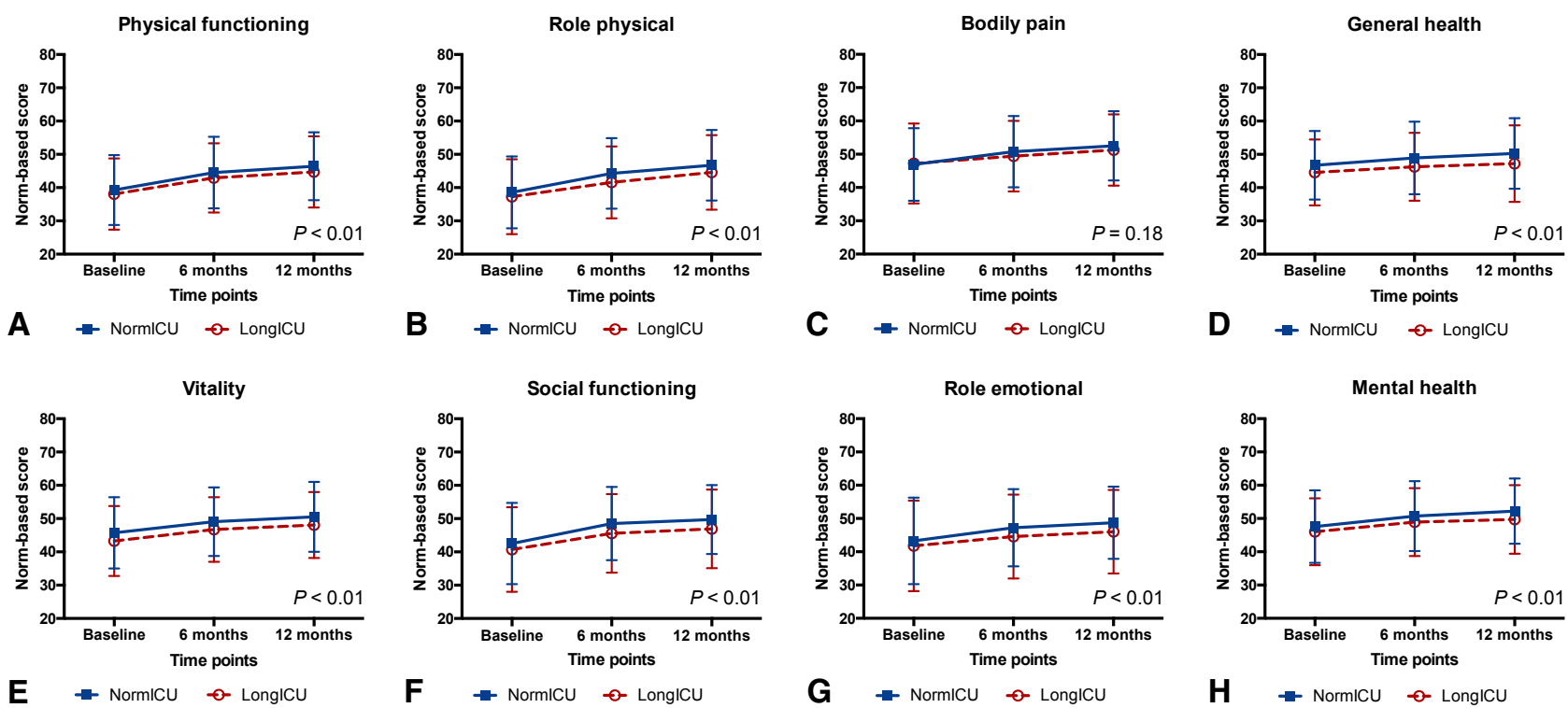

FIGURE E1. Quality of life subdomains at baseline, 6 months, and 12 months after surgery, comparing intensive care unit (ICU) length of stay 0 to 2 days (NormICU), and $\geq 3$ days (LongICU). A, Physical functioning. B, Role physical. C, Bodily pain. D, General health. E, Vitality. F, Social functioning. G, Role emotional. H, Mental health. $P$ is the value for repeated measures analysis of variance between-group analysis over time with Greenhouse Geisser correction. NormICU, Normal intensive care unit stay; LongICU, long intensive care unit stay.

TABLE E1. Reasons for missing Short Form 36 questionnaire (SF36) data

\begin{tabular}{ll}
\hline Follow-up time point & No. of patients completing the SF36 \\
\hline Baseline & $608(100 \% *)$ questionnaire data \\
& $2(\%)$ refused \\
\hline Interim losses $\dagger$ & \\
\hline 6 mo follow-up & $596(93.8 \%)$ complete questionnaire data \\
& $25(4.2 \%)$ patients died \\
& $2(0.3 \%)$ patients withdrew \\
& $1(0.2 \%)$ patient was unable to locate \\
& $594(93.8 \%)$ patients completed the \\
& questionnaire data \\
& $21(3.5 \%)$ patients died \\
\hline
\end{tabular}

*Everyone who accepted to take part completed the SF36 questionnaire. †Interim losses include deaths, withdrawals, and patients who refused to participate in the study. 
TABLE E2. Predicted means of Postoperative Quality of Recovery Scale

\begin{tabular}{|c|c|c|c|c|c|c|}
\hline & $\frac{\mathrm{T1}}{\text { Time point } 1}$ & $\frac{\mathrm{T} 2}{\text { Time point } 2}$ & $\frac{T 3}{\text { Time point } 3}$ & $\frac{T 4}{\text { Time point } 4}$ & $\frac{T 5}{\text { Time point } 5}$ & $\frac{\text { T6 }}{\text { Time point } 6}$ \\
\hline \multicolumn{7}{|c|}{ Overall recovery } \\
\hline NormICU & -5.923 & -2.490 & -2.033 & -0.431 & -0.034 & 0.668 \\
\hline LongICU & -4.532 & -3.216 & -2.433 & -0.984 & -0.349 & -0.086 \\
\hline \multicolumn{7}{|l|}{ Physiological } \\
\hline NormICU & -0.370 & 2.388 & - & - & - & - \\
\hline LongICU & 0.053 & 1.622 & - & - & - & - \\
\hline \multicolumn{7}{|l|}{ Nociceptive } \\
\hline NormICU & -1.44 & -0.128 & -0.184 & 1.253 & 1.858 & 2.426 \\
\hline LongICU & -0.995 & -.0418 & 0.039 & 1.129 & 1.823 & 2.306 \\
\hline \multicolumn{7}{|l|}{ Emotive } \\
\hline NormICU & 1.26 & 1.924 & 1.456 & 1.898 & 1.703 & 2.178 \\
\hline LongICU & 0.269 & 0.944 & 0.402 & 0.921 & 1.337 & 1.639 \\
\hline \multicolumn{7}{|l|}{ Cognitive } \\
\hline NormICU & -2.276 & 0.533 & 2.313 & 3.483 & 3.893 & 4.187 \\
\hline LongICU & -2.351 & -1.028 & 0.705 & 1.923 & 2.389 & 2.972 \\
\hline \multicolumn{7}{|c|}{ Activity of daily livings } \\
\hline NormICU & -1.542 & -0.406 & 1.903 & 3.191 & 3.382 & 3.484 \\
\hline LongICU & -1.562 & -1.109 & -1.027 & 1.595 & 1.749 & 1.923 \\
\hline
\end{tabular}

NormICU, Normal intensive care unit stay; LongICU, long intensive care unit stay.

TABLE E3. Back-transformed means on the Postoperative Quality of Recovery Scale

\begin{tabular}{|c|c|c|c|c|c|c|}
\hline & Time point 1 & Time point 2 & Time point 3 & Time point 4 & Time point 5 & Time point 6 \\
\hline \multicolumn{7}{|c|}{ Overall recovery } \\
\hline NormICU & 0.003 & 0.077 & 0.116 & 0.394 & 0.492 & 0.661 \\
\hline LongICU & 0.011 & 0.039 & 0.081 & 0.279 & 0.414 & 0.479 \\
\hline \multicolumn{7}{|l|}{ Physiological } \\
\hline NormICU & 0.409 & 0.916 & - & - & - & - \\
\hline LongICU & 0.512 & 0.835 & - & - & - & - \\
\hline \multicolumn{7}{|l|}{ Nociceptive } \\
\hline NormICU & 0.191 & 0.468 & 0.454 & 0.778 & 0.865 & 0.919 \\
\hline LongICU & 0.270 & 0.397 & 0.510 & 0.756 & 0.861 & 0.904 \\
\hline \multicolumn{7}{|l|}{ Emotive } \\
\hline NormICU & 0.780 & 0.872 & 0.811 & 0.870 & 0.846 & 0.898 \\
\hline LongICU & 0.567 & 0.720 & 0.600 & 0.715 & 0.792 & 0.837 \\
\hline \multicolumn{7}{|l|}{ Cognitive } \\
\hline NormICU & 0.093 & 0.630 & 0.910 & 0.970 & 0.980 & 0.985 \\
\hline LongICU & 0.087 & 0.263 & 0.670 & 0.872 & 0.916 & 0.951 \\
\hline \multicolumn{7}{|c|}{ Activity of daily livings } \\
\hline NormICU & 0.156 & 0.602 & 0.878 & 0.922 & 0.952 & 0.952 \\
\hline Long ICU & 0.161 & 0.340 & 0.633 & 0.796 & 0.838 & 0.838 \\
\hline
\end{tabular}

NormICU, Normal intensive care unit stay; LongICU, long intensive care unit stay. 
TABLE E4. Incidence of complications for the whole series during hospital stay and at follow-up

\begin{tabular}{|c|c|c|c|c|c|}
\hline \multirow[b]{2}{*}{ Complication } & \multicolumn{5}{|c|}{ Overall series $(n=608)$} \\
\hline & $\overline{\text { Total events }}$ & Intensive care unit & Ward & $6 \mathrm{mo}$ & $12 \mathrm{mo}$ \\
\hline Overall & 309 & $53(8.7)$ & $137(22.5)$ & $29(4.8)$ & $12(2)$ \\
\hline Gastrointestinal & 26 & $6(1)$ & $4(0.7)$ & $11(1.8)$ & $5(0.7)$ \\
\hline Permanent pacemaker & 20 & $0(0)$ & $10(1.6)$ & $4(0.7)$ & $6(1)$ \\
\hline Respiratory & 233 & $67(11)$ & $61(10)$ & $39(6.4)$ & $12(2.1)$ \\
\hline Superficial skin infection & 47 & $5(0.8)$ & $17(2.8)$ & $23(3.8)$ & $2(0.3)$ \\
\hline CVA/TIA & 38 & $17(2.8)$ & $9(1.5)$ & $7(1.1)$ & $5(0.8)$ \\
\hline Vascular & 14 & $0(0)$ & $10(1.6)$ & $1(0.2)$ & $3(0.5)$ \\
\hline Tracheostomy & 8 & $8(1.3)$ & - & - & - \\
\hline AKI (stage I) & 17 & $14(2.3)$ & $3(0.5)$ & - & - \\
\hline AKI (stage III) & 28 & $26(4.3)$ & $2(0.3)$ & - & - \\
\hline Resternotomy & 25 & $23(3.8)$ & $2(0.3)$ & - & - \\
\hline Readmission & 181 & $6(1)$ & $175(28.8)$ & - & - \\
\hline New postoperative hemofiltration & 26 & $23(3.8)$ & $3(0.5)$ & - & - \\
\hline
\end{tabular}

Values are presented with n (\%). CVA, Cerebrovascular accident; TIA, transient ischemic attack; $A K I$, acute kidney injury. 